\title{
REVIEWS
}

\section{Mobility and Cognition in Seniors. \\ Report from the 2008 Institute of Aging (CIHR) Mobility and Cognition Workshop}

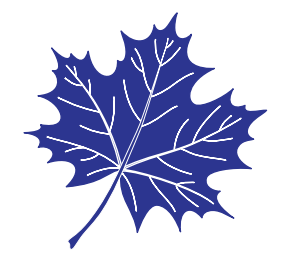

Manuel Montero-Odasso, MD, PhD, AGSF, FRCPC ${ }^{1,2,3}$, Louis Bherer, MPS, $\mathrm{PhD}^{4}$, Stephanie Studenski, MD, MPH, AGSF${ }^{5}$, Karen Gopaul, $\mathrm{MSc}^{1}$, Afua Oteng-Amoako, oD, $\mathrm{MPH}, \mathrm{MSc}^{1}$, Sarah Woolmore-Goodwin, $\mathrm{MSc}^{2}$, Paul Stoole, $\mathrm{PhD}^{6}$, Jennie Wells, MD, FRCPC, FACPA ${ }^{2}$, Timothy Doherty, MD, PhD, FRCPC ${ }^{6}$, Aleksandra A. Zecevic, $\mathrm{PhD}^{7}$, David Galinsky, $\mathrm{MD}^{8}$, R. Jane Rylett, PhD, FCAHS ${ }^{9}$, Jeffrey Jutai, $\mathrm{PhD}^{10}$, Susan Muir-Hunter, $\mathrm{PT} \mathrm{PhD}^{1,7}$, Mark Speechley, $\mathrm{PhD}^{3}$, Richard Camicioli, MD, $\mathrm{FRCPC}^{11}$

${ }^{I}$ Gait and Brain Lab, Parkwood Institute and Lawson Health Research Institute, University of Western Ontario, London, ON; ${ }^{2}$ Division of Geriatric Medicine, Schulich School of Medicine \& Dentistry, The University of Western Ontario, London, ON; ${ }^{3}$ Department of Epidemiology and Biostatistics, University of Western Ontario, London, ON; ${ }^{4}$ Department of Psychology and PERFORM Center, Concordia University, Montreal, QC; ${ }^{5}$ Translational Gerontology Branch Longitudinal Studies Section, National Institute on Aging, Bethesda, MA, USA; ${ }^{6}$ Department of Physical Medicine and Rehabilitation, Schulich School of Medicine \& Dentistry, University of Western Ontario, London, ON; ${ }^{7}$ School of Health Studies, University of Western Ontario, London, ON; ${ }^{8}$ Ben-Gurion University of the Negev, Beer-Sheva, Israel; ${ }^{9}$ Department of Physiology and Pharmacology, Schulich School of Medicine \& Dentistry, University of Western Ontario, London, ON; ${ }^{10}$ Faculty of Health Sciences, Interdisciplinary School of Health Sciences, University of Ottawa, Ottawa, ON; ${ }^{11}$ Faculty of Medicine and Dentistry, Department of Medicine, Division of Neurology, University of Alberta, Edmonton, AB

DOI:http://dx.doi.org/10.5770/cgj.18.188

\section{ABSTRACT}

\section{Background}

The annual Scientific Meeting of the Canadian Association on Gerontology was held on October 24 and 25, 2008 in London, Ontario. Prior to the annual meeting, mobility and cognition experts met on October 23, 2008 to engage in a pre-conference workshop.

\section{Methods}

Discussions during the workshop addressed novel areas of research and knowledge and research gaps pertaining to the interaction between mobility and cognition in seniors.

\section{Results}

Workshop presenters moved from the neuromuscular, biomechanics, and neurology of gait impairments, and falls through the role of cognition and mood on mobility regulation to the whole person in the environment. Research gaps were identified.

\section{Conclusions}

Despite a consensus that mobility and cognition are increasingly correlated as people age, several gaps in our understanding of mechanisms and how to assess the interaction were recognized. The gaps originally identified in 2008 are still pertinent today. Common and standardized assessments for "mobility and cognition" are still not in place in current practice. Interventions that target mobility and cognitive decline as a single entity are still lacking.

Key words: mobility, executive function, dual-task gait, exercises, gait variability, falls, MCI, Parkinson's disease

\section{INTRODUCTION}

"By bringing his body up into a vertical position, modifying his hands in one way and his feet in another, and by improving his brain still further and using it as hard as he could, he stood a chance of success."

-Desmond Morris, “The Naked Ape": A Zoologist's Study of the Human Animal. McGrawHill, 1967, p.25. 
The development of human intelligence is marked by the evolutionary achievement of bipedalism and encephalization. Bipedalism played an essential role in enabling the process of encephalization. As described by Desmond Morris, the achievement of upright walking allowed ancient apes to use their hands during locomotion, an essential step for the creation of hand tools and hunting weapons. This also spurred the subsequent incorporation of more protein into the diet of Homo sapiens, which ultimately contributed to develop larger and complex brains.

Upright bipedal walking and subsequent encephalization are complex features in human evolution and are quintessential hallmarks of the human body. ${ }^{(1)}$ Consequently, and not surprisingly, the functional decline associated with aging originates from two sophisticated and complex human achievements: cognition and gait. ${ }^{(2)}$ As we age, two major geriatric syndromes, cognitive impairment and mobility limitations, become prevalent and burden our quality of life. Extreme manifestations of these syndromes are dementia and falls. Interestingly, these manifestations often coexist in elderly people; dementia affects $30 \%$ of Canadians over age 80 , and falling is a common geriatric syndrome affecting $30 \%$ of older adults over age 65 each year. ${ }^{(3)}$

Evidence shows that cognition and mobility are intertwined. ${ }^{(4)}$ Elderly people with cognitive problems have been found to have a 10 to 15 times greater risk of developing Alzheimer's disease (AD), as well as a higher risk of mobility decline and falling, compared to cognitively normal seniors. ${ }^{(5-7)}$

Cognitive function plays a key role in the regulation and control of even routine walking, especially in older adults. Attention is a necessary cognitive resource for maintaining normal walking and navigation, and attentional deficits are independently associated with postural instability, impairment in performing activities of daily living, and future falls. ${ }^{(8)}$ The role of cognition in walking is ultimately more marked in people with cognitive problems. ${ }^{(9,10)}$

In order to untangle this interesting relationship, we were fortunate to have local and international experts in the field who presented their innovative research on mobility and cognition. The main focus of the workshop was to describe and better understand the interaction between gait and cognition, which may point to new strategies and interventions that delay mobility dysfunction and cognitive decline. Speakers' presentations ranged from the epidemiology and cellular mechanisms related to mobility, muscle function and neurocognitive control of mobility, through to neuromuscular biomechanics and the neurology of falls; finally, to the whole person in the environment including intelligent assistive devices.

This workshop provided a seminal forum in which research on "mobility and cognition" in older adults was examined with key experts in the field. Several important research gaps were identified and the clear necessity for the translation of research knowledge to levels of care was emphasized. Despite research progress in the areas of "mobility and cognition" in older adults since this 2008 workshop, the research gaps identified then remain highly relevant today.

\section{Background}

Over the next 20 years there will be a substantial increase in the number of Canadians aged 65 and older. ${ }^{(11)}$ Increased life expectancy is often accompanied by disability, stemming in a large part from two major geriatric syndromes: mobility and cognitive impairment. It has been demonstrated that the decline in gait velocity is a preclinical marker of diseases. ${ }^{(12-}$ 15) Similarly, aging is associated with decreases in cognitive function and the development of dementia. Eight per cent of Canadians aged 65 and older meet the criteria for dementia, and this prevalence increases to $35 \%$ in people over the age of $85 .^{(16-18)}$ Furthermore, mobility and cognitive impairment appear to become more related as people age. ${ }^{(19-21)}$ Specifically, gait slowing and dementia often co-exist in the same individual, have been attributed to changes in specific brain regions, ${ }^{(22-25)}$ and may contribute to an increased risk of falls and disability. ${ }^{(26,27)}$

\section{Workshop Themes}

- The science underlying connections of mobility and cognition

- Clinical and research perspectives on mobility and cognition

- Interventions for mobility and cognition as a single entity

\section{Objectives}

1. Provide an overview of the state of understanding and research in mobility and cognition;

2. Explore the value of the dual-task gait as a way to evaluate cognitive and mobility relationships;

3. Investigate interventions to improve mobility and cognitive impairments as a solo entity in seniors; and

4. Identify the knowledge gaps and strategic research priorities in mobility and cognition, in support of the Mobility in Aging initiative from the CIHR, Institute of Aging.

\section{Participants}

There were 70 workshop participants and 12 speakers and moderators. Participants were researchers, physicians, and allied health clinicians with expertise in elderly care. For a list of speakers and moderators, and an evaluation summary of the workshop, see Appendix A.

\section{RESEARCH PRESENTATIONS AND SPEAKERS}

The workshop was opened with welcome remarks by Dr. Jane Rylett of the University of Western Ontario and Chair 
of CIHR-IA Advisory Board who highlighted the research priority on mobility and aging as part of the CIHR Institute of Aging. Dr Manuel Montero-Odasso moderated the workshop. The following are summaries of the research presentations in order of presentation.

\section{Epidemiology of the relationship between mobility and cognition. Is mobility a predictor of cognitive decline? \\ Dr. Stephanie A. Studenski, University of Pittsburgh, Pittsburgh, PA, USA}

Among older adults, cognitive and movement disorders co-occur more frequently than would be expected by chance, and the presence of either disorder increases the risk of onset of the other. Epidemiological evidence supports associations between cognition, movement and mood both cross-sectionally and longitudinally. Additionally, cognition, movement, and mood are associated with identifiable brain abnormalities such as cerebral white matter disease. ${ }^{(28)}$ In a model proposed by Dr. Studenski's group, regions of ischemia or leukoariosis in white matter or atrophy in regions of grey matter can result in psychomotor slowing which culminates in disorders of cognition, movement, and mood that ultimately lead to functional decline and falls. Since the decline in cognition and depressive symptoms are both associated with slower gait and falls, future studies of aging should include combined measures of movement, cognition, and mood, as should prevention and intervention studies. For future research, animal model studies could help us better understand the mechanism behind these associations.

\section{Neuromuscular changes associated with poor mobility and aging. Dr. Timothy Doherty, Western University, London, ON, Canada}

Aging is associated with substantial decline in neuromuscular function. This is best characterized by loss of muscle mass and strength in distal and proximal muscles in the upper and lower limbs - a phenomenon referred to as "sarcopenia". $(29,30)$ Aging is also associated with significant differences in the contractile properties of skeletal muscle, characterized by slowing for both contraction and relaxation speeds. Peripheral fatigue may be somewhat less in the older adult's skeletal muscle, particularly when measured relative to maximal force values. The underlying cause of sarcopenia is multifactorial, but age-associated loss of motor units with incomplete re-innervation appears to be a major influence. ${ }^{(30)}$ Other factors include disuse, nutritional deficiency, inflammatory effects, and hormonal factors. Neuromuscular function and sarcopenia have a direct impact on mobility and falls. The loss of strength, and slower contractile speed which results in less power of lower limb muscles, are major factors that contribute to poor mobility in the elderly.

\section{Balance performance, behaviour and cognition. The impact on falls. \\ Dr. Aleksandra Zecevic, Western University Ontario, London, ON, Canada}

Cognitive impairment reduces central nervous system (CNS) ability to process and integrate multiple sensory inputs which results in balance problems, increased postural sway, and slower walking speed; however, causes of falls among cognitively impaired individuals are still poorly understood. A Seniors Falls Investigative Methodology (SFIM) was created to investigate falls in seniors which shifted the focus from the faller to the system in which the person functions. ${ }^{(31,32)}$ Studies in a community, hospitals, and long-term care facilities identified psychological contributors to falls that include poor decision making, cognition, and attention. One way to manage and prevent falls and injuries in individuals with dementia is to accept fallers as they are and build a safe system that will accommodate them. This requires removing preconditions that lead to falls, improving and optimizing formal and informal supervision, and establishing safety barriers through policies and practices in all organizations involved in care of patients with cognitive impairments. System-wide interventions and safety improvements should be integrated into health-care organizations and the community in order to ensure safe mobility in elderly Canadians.

\section{Assistive devices for mobility and cognition. Can we have a unified approach? The role of cognition in assistive technology for mobility. Dr. Jeffrey Jutai, University of Ottawa, Ottawa, ON, Canada}

Assistive devices, such as canes, walkers, and wheelchairs, are very commonly used with older patients who have mobility difficulties. ${ }^{(33)}$ In recommending devices, clinicians attend first to the physical capabilities and limitations of their patients. Evidence indicates that impaired cognitive processes, particularly cognitive flexibility and working memory, are prevalent in older people and associated with falls. Impaired cognition affects proper utilization of mobility devices and increases the risk of falls due to reduced attentional capacity, impaired central processing and integration, impaired judgment, and self-regulation and execution of postural responses, resulting in impaired balance, gait, anticipatory postural adjustments, and reactive postural responses. The effects of patients' cognition on decisions about mobility devices are poorly understood. Successful device-assisted rehabilitation and management of mobility difficulties may require strategies that target both physical functions and cognitive processes. Important research questions that address gaps in this area include: 1) Are elderly rehabilitation patients with impaired executive-controlled processes significantly more likely to report problems with the safety and effective use of mobility 
devices? 2) Is cognitive impairment reliably correlated with the decision to recommend or not the use of mobility assistive devices? 3) Do the relationships among cognition, mobility, and device use change predictably over time? 4) What are the opportunities for improving the usefulness of theory-derived cognitive assessments in making decisions about the selection of mobility assistive devices?

\section{History of the mobility and gait analysis in geriatric research. Dr. David Galinsky, Ben Gurion University, Be'er Sheva, Israel}

Prof. Bernard Isaacs established the concept of the "giants" in geriatric medicine: Immobility, Instability, Intellectual, and Incontinence. ${ }^{(34)}$ One of the main negative outcomes of the giants is falls. Gait assessment procedures used to detect risk of falls have required considerable investment in equipment for sophisticated biomechanical testing, limiting the use of gait testing procedures to the laboratory or clinics. We pioneered a simple gait assessment using an effective method for home and community assessment: the ELGAM (extra-laboratory gait assessment method). ${ }^{(35,36)}$ A study was conducted on residents of a sheltered housing for seniors ranging in age from 63-90 years. A complete geriatric comprehensive assessment was performed and a neurological and physical exam that included the ELGAM was administered. (37) Through ELGAM, slow walking, small steps, impaired balance, and difficulty turning the head were all significantly associated with unstable gait and, therefore, falls. The ELGAM was an efficient and effective method of recording gait and the early detection of risk of falls.

\section{Gait impairment and Parkinsonism:}

Risk factors for cognitive decline \& dementia. Dr. Richard Camicioli, University of Alberta, Edmonton, AB, Canada

Mild Parkinsonism and gait impairment occur prior to the development of mild cognitive impairment, which is a precursor to dementia. Age-related gait changes may be explained by co-morbid conditions, including vascular disease, which increases the risk of cognitive decline and dementia. However, cognitive and mobility changes can be part of the spectrum of degenerative disease in the absence of illness or frailty and, in some cases, remain unexplained. Longitudinal studies can help determine if specific aspects of cognitive decline are related to Parkinsonism and gait impairment. Indeed, the pathology of age-related gait impairment was shown to correlate with nigral neurofibrillary tangle count, while Parkinson's showed similar correlations. Specific degenerative diseases associated with aging, such as dementia with Lewy bodies and Parkinson's disease, are also characterized by Parkinsonism and are associated with cognitive decline and gait impairment. ${ }^{(38-40)}$
Gait and dual-tasking. Is it the new paradigm to explore mobility and cognition in older people? Dr. Manuel Montero-Odasso, Western University, London, ON, Canada

A seminal study, "stop walking while talking", demonstrated that the inability to maintain a conversation while walking is a marker for future falls in older adults. ${ }^{(41)}$ Walking while performing a secondary task (dual-task paradigm) has become the classic way to assess the interaction between cognition and gait. Previous research has described the effect of dualtasking on gait performance, showing specific associations between slowing gait and executive dysfunction and attention deficits. Results from our "Gait and Brain Study" demonstrate that impairments in specific cognitive domains - attention, executive function, and working memory - are associated with slow gait velocity and slower gait velocity under dualtask conditions, and higher variability indicating that those specific cognitive domains are crucial for maintaining normal gait performance. ${ }^{(42,43)}$ Therefore, gait variability under dual-tasking may be a sensitive measure of Mild Cognitive Impaired seniors at risk of developing dementia and risk of falling. ${ }^{(9)}$

Effect of exercise on cognition in older adults. Dr. Louis Bherer, Université de Québec à Montréal et Institut universitaire de gériatrie de Montréal, Montreal, QC, Canada

An increasing number of studies suggest that physical activities might protect against age-related cognitive decline. In fact, aerobic training leads to a significant improvement in $\mathrm{VO} 2{ }_{\text {max }}$ that correlates with increased cognitive performances in sedentary older adults, ${ }^{(44)}$ with larger benefits on attentional control functions, as required, to share attention between multiple tasks. To further assess the impact of physical activity on cognitive performances in older adults, we conducted cross-sectional and intervention studies involving various populations of older adults, from sedentary and frail older adults ${ }^{(45,46)}$ to athletes or highly active individuals who engage in intense fitness training programs. ${ }^{(45)}$ In our cross-sectional study we found that being physically active can protect against decline in cardiorespiratory fitness, as well as less of a decline in dual task performance. ${ }^{(46)}$ Our intervention studies, designed for sedentary older adults, demonstrate that just three months of physical exercise can improve physical health and attentional control functions in older adults and even frail older adults. ${ }^{(45)}$

\section{Tai Chi as an intervention on cognition. A review of the evidence. Dr. Jennie Wells, Western University, London, ON, Canada}

Tai Chi is growing in popularity in western culture as a form of exercise, relaxation and health therapy. It has been shown 
to improve cardiovascular fitness, blood pressure, immunity, balance, and reduce falls, LDL cholesterol, pain, anxiety, and depression. Tai Chi may also benefit cognition since it is a form of aerobic exercise and reduces depression and stress, both of which impair cognition. However, a wider variety of research strategies are needed to support evidence that Tai Chi is beneficial for cognition. Designing research studies that involve Tai Chi as an intervention can be challenging since it is a multi-component pluralistic intervention which can vary, depending on individual participation, duration of practice, and frequency. A "whole system" approach that recognizes the complex interactions of Tai Chi and utilizes multiple disciplines to conduct and analyze the data would be the best approach.

\section{Round Table: Mobility and Cognition in Older Adults}

Several research gaps were identified from the workshop and were grouped under five themes. Future actions discussed were also included in each theme. A synopsis including key points, main gaps, and future directions addressed during the round table is summarized in Figure 1.

\section{Understanding the neurobiology of mobility decline}

When examining the neurobiology of mobility decline and falls, the panel noted pre-existing knowledge that ageassociated muscle loss (sarcopenia), deficits in neural control of gait and balance, chronic nutrient deficiencies (including Vitamin D), polypharmacy and impaired cardiovascular regulation (affecting postural control of blood pressure) are important contributors to falls among older people. Age-related decline is multi-factorial, suggesting a single problem identification is not likely to be useful for diagnosis or detection of falls. Dr. Doherty noted that sarcopenia is not necessarily correlated with the risk of falling; rather, it is neuromuscular function (strength, power, balance) in combination with sarcopenia that appears to have a direct impact on mobility and falls. ${ }^{(47)}$ The interaction of these two phenomena needs to be further explored to better understand challenges in mobility in later life. The role of the CNS, including the integration of sensory inputs, in mobility decline and falls is an emerging area of research. As elderly people continue to age, they become more dependent on their visual cues and less reliant on other senses. These sensorial changes have negative consequences on the neural regulation of mobility as seniors on average have poorer vision and vestibular impairments, which affect the regulation of their balance and mobility. Dr Montero-Odasso highlighted that dual-task gait is a paradigm which can help to evaluate the effect of cognitive resources on mobility and gait performance. Recently, it has become evident that higher level cognitive functions also have a critical role in the regulation of routine walking. Falling itself is not only an issue of the physical mechanics of movement,
1. Cognition and mood are key contributors of the age associated mobility decline.

2. Cognition and Mobility need to be seen as combined clinical/ research entity rather than separate problems.

3. Research in mobility should incorporate measures and outcomes related to cognition and vice versa.

4. Common language and common standardized assessments between researchers in the fields of mobility and cognition are needed. Evidence-based findings must be standardized.

5. Earlier detection, intervention, and assessment of cognitive and mobility decline in seniors are necessary.

6. Gait velocity and dual-task gait tests can be used as instruments to detect early changes in cognitive and mobility decline. Further consensus in measurements is needed.

7. The interaction between neuromuscular and brain function in combination with sarcopenia needs to be better explored to understand challenges to mobility in later life.

8. Interventions should have targets not only in particularly structural brain changes after exercises intervention, but also across the bio-psycho-social domain.

9. Future research that uses a unified approach of physical performance measurements, cognitive measures, and neuroimaging is needed and may be most feasible through the effective collaboration across disciplines.

10. Exclusive focus on the person who falls is not enough. Contributions of the system in which the faller operates must be identified and addressed.

FIGURE 1. Key points, gaps encountered, and future actions discussed in the workshop

but the failure of a dual-process that requires thinking about movement and then performing the movement in the context of navigation through the environment.

\section{The effect of physical exercise on cognition in vulnerable populations}

We are still not fully aware of the mechanisms by which physical activity and exercise influences cognition, and research in this area is just emerging. Consequently, we were not able to identify the best protocol or recommendations for prescribing exercise interventions for preventive purposes or know for whom such preventions might positively enhance cognitive performance. Moreover, research studies targeting vulnerable populations, such as those with mild cognitive impairment (MCI), are lacking. Previous work has established that physical activity protects against cognitive decline. In corroboration, Dr. Bherer presented data from studies indicating better cardiorespiratory fitness (measured as oxygen consumption $\left(\mathrm{VO}_{2 \max }\right)$ is predictive of cognitive performance 
over a six-year period of follow up. Similarly, interventional studies in which participants are assigned randomly to aerobic training (e.g., walking and/or biking) suggest cardiorespiratory fitness plays a causal role in building cognitive reserves.

Future research should focus on the effects of different exercise programs on cognitive domains such as attention and memory, especially because attention and executive function deficits have been strongly associated with risk of falling. This approach will also advance preventive practice if physical fitness measures are considered in the assessments of falls risk.

\section{Interrelation and interventions for cognition, mobility and mood}

While the panel during the roundtable determined there should be a concerted movement toward understanding the central neural basis of mobility, particularly structural and functional brain changes after exercise interventions, it was proposed that interventions should have targets on the bio-psycho-social domain. This will require identification of contributing factors to falls beyond the capacities and characteristics of the person who fell, as described by SFIM.

Dr. Wells demonstrated from her literature review that implementation of Tai Chi into a senior's daily routine yields improvements in muscle strength, balance, and mood. Other types of interventions, such as seen in research by Dr. Montero-Odasso, show that nutritional supplements such as vitamin $\mathrm{D}$, which targets the brain and skeletal muscle, can be useful interventions for improving mobility in older adults with vitamin $\mathrm{D}$ deficiency. ${ }^{(48)}$

While emerging evidence indicates physical activity, exercises, and balance training reduce the risk of falls, improve cognition, and enhance dual-tasking, clinicians are finding barriers to adopt this intervention into their practice. It was concluded that more needs to be done to ensure knowledge based on research evidence becomes implemented in clinical practice. Consensus statements and clinical guidelines may help to translate the evidence from research studies to daily practice.

\section{Scientific design, level of analysis, and common measures}

Presenters agreed on the need for a consensus on how to design future research on mobility and cognition. Cognitive and mobility decline are multi-factorial problems which can be addressed at different levels and with both quantitative and qualitative methodologies.

Future research on the longitudinal pathophysiology should include intermediate outcomes as proxies of mobility and cognition that lead to falls to understanding potential mechanisms. Case-study approach to investigations of system-wide true causes of falls, proposed by Dr. Zecevic, is of special interest for falls in individuals with advanced cognitive impairment.
Unified approaches to understanding the relationships among mobility, cognition, and mood are scarce. Because mobility and cognitive decline are complex phenomena, multi- or trans-disciplinary approaches will likely be the most effective. Such studies would include measures of mobility, cognition, and mood in the same design rather than treating them as unrelated outcomes. Future research that uses physical performance measurements, cognitive measures, and neuroimaging may also help to overcome this challenge. Work in this area will require effective collaborations across disciplines. One of the main gaps identified was the lack of a common language and standardized assessments between researches in the fields of mobility, cognition, and mood.

\section{Determining the most appropriate approach for early detection}

Early detection of risk was another gap that emerged from the day's discussions. Dr. Montero-Odasso postulated that gait performance can be seen as a final common pathway that integrates several systems including the cardiovascular, neuromuscular, and neurological. Accordingly, gait assessment can be utilized as a screening tool to detect those older individuals at higher risk of mobility and cognitive decline. A wealth of evidence supports that gait velocity and dual-task gait tests can be used as instruments to detect early changes in cognitive and mobility decline. However, there is no consensus on which is the ideal gait screening measure. A key gap in current research is that we do not have an accepted standard measure of mobility impairment and fall risk that will accommodate the heterogeneity of the older population.

\section{Future Directions}

"If we look ten years ahead, what strategies should we consider toward advancing this knowledge from a Canadian perspective? Do we look at mechanisms, interventions, neural correlates? What will make a difference for our seniors and the way we treat, assess, and prevent mobility and cognition decline?"

The panel's unanimous response to this question, raised by Dr Michael Borrie (Western University), was that novel techniques, such as new developments in brain imaging and quantitive motion/gait analysis, will likely advance our understanding of the physiological basis of the relationship between gait, mood, and cognition. It was also suggested that the different bio-psycho-social research areas should not be seen as competing interests, but be integrated into future research approaches. Each domain must be cared for, as the basis for patient/resident-centered focus and care. The most beneficial way forward would be to determine closely related areas and study them together in order to observe their critical interactions. 
Dissemination of the knowledge from researchers and experts should be more effectively applied to clinical practice. This is a serious challenge in the care of the elderly. Ideally, front-line staff, as experts working in the field, will be included in research collaboration from the conceptualization of new research projects.

In the future, our health-care system will become increasingly taxed with the influx of seniors and for the disability stemming from cognitive and mobility declines. To circumvent this mounting stress on seniors and the health-care system, we need to support the creation of a culture that enables people of all ages to exercise both the mind and the body.

At a social and governmental level, we would need to educate policy makers about the needs for - and benefits of — preventing health decline before it becomes unmanageable at any level of care provision.

\section{CONCLUSIONS AND REVISITING THE WORKSHOP SIX YEARS LATER}

This workshop provided a seminal environment in which research on "mobility and cognition" in older adults was examined with key Canadian and international experts. Several important research gaps were identified and the clear necessity for the translation of research knowledge at primary care levels was emphasized. Despite research progress in the areas of "mobility and cognition" in older adults since this 2008 workshop, the research gaps identified remain extremely relevant today.

One of the main gaps identified - the lack of a common language and standardized assessments between researches in the fields of mobility, cognition, and mood - still needs to be overcome to better coordinate research and communicate results. This workshop served to seed an initial Pan-Canada collaboration which was crystalized in 2012 in the Mobility, Exercise and Cognition team aggregated under the Canadian Consortium in Neurodegeneration and Aging, funded by CIHR.

Our goal as researchers, community healthcare workers, front-line staff, and physicians is to ensure quality of life and disability free life for our Canadian seniors. This forum was an initial step to ensure action is taken and research is disseminated to those who will benefit from it directly.

\section{ACKNOWLEDGEMENTS}

This workshop would be impossible without the constant assistance of Ms. Sarah Woolmore-Goodwin, research assistant from Parkwood Hospital, and the logistic and intellectual support of Dr. Susan Crawford and Ms. Rowena Tate from Institute of Aging (CIHR). We thank the ARGC-Lawson and its Director, Dr. Rob Petrella for their support with this workshop. Very special thanks to Saskia Sivananthan for her contribution on the workshop report sent to CIHR. Finally, we thank the assistance of Drs. Stole and Petrella as associated moderators.

\section{CONFLICT OF INTEREST DISCLOSURES}

The authors declare that no conflicts of interest exist. This workshop was sponsored by the Institute of Aging (CIHR) and co-sponsored by the Lawson Research Institute (London, Ontario \& Western University). Dr Montero-Odasso's program in "Gait and Cognition" is funded by CIHR, operating grant MOP12243.

\section{REFERENCES}

1. Lewin R, Foley R. Principles of human evolution, 2nd. ed. Malden, MA: Blackwell Publishing, Inc.; 2003.

2. Montero-Odasso M, Hachinski V. Preludes to brain failure: executive dysfunction and gait disturbances. Neurol.Sci. 2014;35(4):601-04.

3. A Profile of Disability in Canada, 2001. Ottawa: Statistic Canada; 2007. Available from: http://www.statcan.gc.ca/pub/89-577-x/ index-eng.htm

4. Montero-Odasso M, Verghese J, Beauchet O, et al. Gait and cognition: a complementary approach to understanding brain function and the risk of falling. J Am Geriatr Soc. 2012;60(11):2127-36.

5. Boyle PA, Wilson RS, Aggarwal NT, et al. Mild cognitive impairment: risk of Alzheimer disease and rate of cognitive decline. Neurology. 2006;67(3):441-45.

6. Muir SW, Gopaul K, Montero Odasso MM. The role of cognitive impairment in fall risk among older adults: a systematic review and meta-analysis. Age Ageing. 2012;41((3)):299-308.

7. Petersen RC. New clinical criteria for the Alzheimer's disease spectrum. Minn Med. 2012;95(1):42-45.

8. Woollacott M, Shumway-Cook A. Attention and the control of posture and gait: a review of an emerging area of research. Gait Posture. 2002;16(1):1-14.

9. Montero-Odasso M, Muir SW, Speechley M. Dual-task complexity affects gait in people with mild cognitive impairment: the interplay between gait variability, dual tasking, and risk of falls. Arch Phys Med Rehabil. 2012;93(2):293-99.

10. Muir SW, Speechley M, Wells J, et al. Gait assessment in mild cognitive impairment and Alzheimer's disease: the effect of dual-task challenges across the cognitive spectrum. Gait Posture. 2011;35(1):96-100.

11. National Advisory Council on Aging. 1999 and beyond: challenges of an aging Canadian society. Ottawa: Industry Canada; 1999.

12. Studenski S. Mobility. In: Hazzard W, Tinetti ME, Blass JP, et al., editors. Principles of geriatric medicine and gerontology, $5^{\text {th }}$ edition. New York: McGraw-Hill; 2003. p. 947-60.

13. Studenski S, Perera S, Wallace D, et al. Physical performance measures in the clinical setting. J Am Geriatr Soc. 2003;51(3):314-22.

14. Montero-Odasso M, Schapira M, Varela C, et al. Gait velocity in senior people. An easy test for detecting mobility impairment in community elderly. J Nutr.Health Aging. 2004;8(5):340-43.

15. Montero-Odasso M, Schapira M, Soriano ER, et al. Gait velocity as a single predictor of adverse events in healthy seniors aged 75 years and older. J Gerontol A Biol Sci Med Sci. 2005;60(10):1304-09. 
16. Canadian study of health and aging: study methods and prevalence of dementia. CMAJ. 1994;150(6):899-913.

17. Third Canadian Consensus Conference on Diagnosis and Treatment of Dementia, Montreal, QC, Mar 9-11 2006. Available from:http:// www.cccdtd.ca/pdfs/final_recommendations_ccedtd_2007.pdf

18. Thomas VS, Darvesh S, MacKnight C, et al. Estimating the prevalence of dementia in elderly people: a comparison of the Canadian Study of Health and Aging and National Population Health Survey approaches. Int Psychogeriatr. 2001;13 Supp 1:169-75.

19. Montero-Odasso M, Casas A, Hansen K, et al. Test-retest reliability of quantitative gait analysis in people with mild cognitive impairment. Can J Geriatr Med Psychiatry. 2007;10(3):33-34.

20. Montero-Odasso M, Bergman H, Phillips NA, et al. The effect of executive and memory dysfunction in gait performance in a cognitive impairment population. J Am Geriatr Soc. 2006;54:S154.

21. Montero-Odasso M. The value of gait velocity test for highfunction populations. J Am Geriatr Soc. 2006;54(12):1949-50.

22. Rosano C, Brach J, Longstreth Jr WT, et al. Quantitative measures of gait characteristics indicate prevalence of underlying subclinical structural brain abnormalities in high-functioning older adults. Neuroepidemiol. 2006;26(1):52-60.

23. Allan LM, Ballard CG, Burn DJ, et al. Prevalence and severity of gait disorders in Alzheimer's and non-Alzheimer's dementias. $J$ Am Geriatr Soc. 2005;53(10):1681-87.

24. Bloem BR, Gussekloo J, Lagaay AM, et al. Idiopathic senile gait disorders are signs of subclinical disease. J Am Geriatr Soc. 2000;48(9):1098-101.

25. Camicioli R, Howieson D, Oken B, et al. Motor slowing precedes cognitive impairment in the oldest old. Neurology. 1998;50(5):1496-98.

26. Campbell AJ, Borrie MJ, Spears GF. Risk factors for falls in a community-based prospective study of people 70 years and older. J Gerontol. 1989;44(5):M112-M117.

27. Tinetti ME, Speechley M, Ginter SF. Risk factors for falls among elderly persons living in the community. $N$ Engl $\mathrm{J}$ Med. 1988;319(26):1701-07.

28. Rosano C, Aizenstein H, Brach J, et al. Special Article: Gait measures indicate underlying focal gray matter atrophy in the brain of older adults. J Gerontol A Biol Sci Med Sci. 2008;63(12):1380-88.

29. Berger MJ, Doherty TJ. Sarcopenia: prevalence, mechanisms, and functional consequences. Interdiscip Top Gerontol. 2010;37:94-114.

30. Doherty TJ. Invited review: Aging and sarcopenia. J Appl Physiol. 2003;95(4):1717-27.

31. Zecevic AA, Salmoni AW, Speechley M, et al. Defining a fall and reasons for falling: comparisons among the views of seniors, health care providers, and the research literature. Gerontologist. 2006;46(3):367-76.

32. Zecevic AA, Salmoni AW, Lewko JH, et al. Seniors falls investigative methodology (SFIM): a systems approach to the study of falls in seniors. Can J Aging. 2007;26(03):281-90.

33. Bateni H, Maki BE. Assistive devices for balance and mobility: benefits, demands, and adverse consequences. Arch Phys Med Rehabil. 2005;86(1):134-45.

34. Isaacs B. The giants of geriatrics. In: The challenge of geriatric medicine. Oxford: Oxford University Press; 1992. p. 1-7.
35. Cwikel JG, Fried AV, Biderman A, et al. Validation of a fall-risk screening test, the Elderly Fall Screening Test (EFST), for community-dwelling elderly. Disabil Rehabil. 1998;20(5):161-67.

36. Muro-de-la-Herran A, Garcia-Zapirain B, Mendez-Zorrilla A. Gait analysis methods: an overview of wearable and non-wearable systems, highlighting clinical applications. Sensors. 2014;14(2):3362-94.

37. Fried AV, Cwikel J, Ring H, Galinsky D. ELGAM-extra-laboratory gait assessment method: identification of risk factors for falls among the elderly at home. Int Disabil Stud. 1990;12(4):161-64.

38. Camicioli R, Howieson D, Lehman S, et al. Talking while walking: the effect of a dual task in aging and Alzheimer's disease. Neurology. 1997;48(4):955-58.

39. Camicioli R, Howieson D, Oken $\mathrm{B}$, et al. Motor slowing precedes cognitive impairment in the oldest old. Neurology. 1998;50(5):1496-98.

40. Camicioli R, Majumdar SR. Relationship between mild cognitive impairment and falls in older people with and without Parkinson's disease: 1-Year prospective cohort study. Gait Posture. 2010;32(1):87-91.

41. Lundin-Olsson L, Nyberg L, Gustafson Y. "Stops walking when talking" as a predictor of falls in elderly people. Lancet. 1997;349(9052):617.

42. Montero-Odasso M, Casas A, Hansen KT, et al. Quantitative gait analysis under dual-task in older people with mild cognitive impairment: a reliability study. J Neuroeng Rehabil. 2009 Sep $21 ; 6(1): 35$.

43. Montero-Odasso M, Bergman H, Phillips NA, et al. Dual-tasking and gait in people with mild cognitive impairment. The effect of working memory. BMC Geriatr. 2009;9:41.

44. Kramer AF, Hahn S, Cohen NJ, et al. Ageing, fitness and neurocognitive function. Nature. 1999;400(6743):418-19.

45. Bherer L, Erickson KI, Liu-Ambrose T. Physical exercise and brain functions in older adults. J Aging Res. 2013;2013:197326.

46. Bherer L, Kramer AF, Peterson MS, et al. Training effects on dual-task performance: are there age-related differences in plasticity of attentional control? Psychol Aging. 2005;20(4):695-709.

47. Visser M, Schaap LA. Consequences of sarcopenia. Clin Geriatr Med. 2011;27(3):387-99.

48. Muir SW, Montero-Odasso M. Effect of vitamin D supplementation on muscle strength, gait and balance in older adults: a systematic review and meta-analysis. J Am Geriatr Soc. 2011;59(12):2291-300.

Correspondence to: Manuel Montero-Odasso, MD, PhD, AGSF, FRCPC, Gait and Brain Lab at the Parkwood Institute and Lawson Health Research Institute, Division of Geriatric Medicine at the Schulich School of Medicine \& Dentistry, and the Department of Epidemiology and Biostatistics, University of Western Ontario, 801 Commissioners Rd. E., Rm A-280, London, ON N6C 5J1, Canada

E-mail: mmontero@uwo.ca 


\section{APPENDIX A}

\section{Workshop members}

This is a complete list of the moderators, speakers, facilitator, and administrators. Dr. Anne Martin-Matthews (Scientific Director of the Institute of Aging CIHR), Rowena Tate and Dr. Susan Crawford (Assistant Director of CIHR-IA), Manuel Montero-Odasso (workshop leader and moderator), Paul Stole and Rob Petrella (associate moderators), Sarah Woolmore-Goodwin (research assistant and administrative support), Dr. Jane Rylett (welcome remarks), Dr. Stephanie A. Studenski (speaker), Dr. Timothy Doherty (speaker), Dr. Aleksandra Zecevic (speaker), Dr. Jeffrey Jutai (speaker), Dr. David Galinsky (speaker), Dr. Richard Camicioli (speaker), Dr. Louis Bherer (speaker), and Dr. Jennie Wells (speaker).

\section{Workshop Evaluation}

The workshop evaluation was positive, with the majority of participants indicating that the workshop was a successful and worthwhile event. A quantitative anonymous evaluation conducted by the staff from CIHR-IA determined that $85 \%$ of the participants found that most of the information provided was novel to them. Eighty-three per cent of the participants also attributed the success of the workshop to the quality, diversity, and intellectual range of participants who attended the workshop. The workshop was viewed as an "excellent overview of wide ranging work in an exciting research area with major implications for clinical practice, environmental design, and quality of life for older persons."

Respondents reported that $90 \%$ of the information was useful. The dynamic of the group interaction and round-table discussion was a highlight for the respondents and the engagement with the experts was applauded. "There was great breadth and depth in each of the speakers discussions" reported one student workshop participant, and this sentiment was shared among most respondents. Overall, the workshop was a successful day of learning, sharing, and building knowledge together. It was a novel creative process where community and industry, science and clinical experience collaborated and worked towards developing a plan to create intervention for future populations of seniors while assisting those who are currently afflicted with mobility and cognition deficits. 